\title{
Editorial:
}

\section{How Do We Go About Understanding the Nature of Nonlinear Learning?}

\author{
Jia Yi Chow ${ }^{1, *}$ and Yeou-Teh Liu ${ }^{2}$
}

\author{
${ }^{1}$ Physical Education and Sports Science, National Institute of Education, Nanyang Technological University, Singapore \\ ${ }^{2}$ National Normal Taiwan University, Taiwan
}

It seems strange to begin this special issue on nature of nonlinear learning with a discussion on how do we go about understanding nonlinear learning. But true to the theme of this special issue, nothing ever really works in a fully linear fashion! There are certainly many ways to understand nonlinear learning and its complexity. But, by beginning with the 'how to understand question', it provides a clear sign-post to what we want to achieve with this special issue. It sets the stage for experts in nonlinear learning to then answer the 'what' (what is nonlinear learning?), 'how' (how to examine nonlinear learning?), 'why' (why is nonlinear learning possible?) and the 'when and where' (when and where to apply features of nonlinear learning?) questions. By asking these simple questions that serve as the pillars for framing this special issue, we hope to provide a comprehensive discussion on understanding the nature of nonlinear learning.

While we believe in nonlinearity, unfortunately the nature of the journal platform to share content is constrained to a more linear approach. Although we have identified the different questions that should be asked to guide understanding, we have to begin from somewhere and we have identified four major sections of discussion to help our readers to access the content for this special issue.

The first section shares valuable insights on the theoretical grounding for understanding the features of nonlinear learning. Newell and Liu present an insightful discussion on the functions of learning and the acquisition of motor skills with special reference to sport. Particularly, the suggestion is that the functions of learning can be dependent on the task to be learnt. This is followed by Davids' paper on explaining how the learner is viewed as a nonlinear dynamical system and the appropriate learning designs to enhance nonlinear learning. These two papers provide a strong theoretical discussion on nonlinear learning and are suitably positioned to answer the key question of, 'What is the nature of nonlinear learning?'

While theoretical conceptualisations are critical in providing the framework to undertake empirical investigations, the availability of the right analytical tools is pertinent to

*Address correspondence to this author at the Physical Education and Sports Science, National Institute of Education, Nanyang Technological University, Singapore ; Tel: (65) 6790-3692; Fax: (65) 6896-9260;

E-mail: jiayi.chow@nie.edu.sg ensure that the empirical data used to examine nonlinear learning is relevant. To meet this aim, Rein provides a sound discussion on some current and innovative analysis methods to examine empirical investigations on nonlinear learning and behaviours. The information shared should provide a starting point for researchers who are interested in understanding the learning and performance processes from a nonlinear perspective. Rein's paper appropriately addresses the question of 'how to examine nonlinear learning?'

Empirical investigations have been undertaken by numerous researchers in recent years and a few are shared here to propose that nonlinear learning is possible. Button and colleagues provide an interesting review on various empirical works on nonlinear learning and is therefore aptly positioned to lead the discussion for this section. Edwards and Hodges subsequently reports an interesting investigation on the role of variability in enhancing learning and performance. Based on their discussion, it seems that while variability has the potential to bring about positive change to learning and performance, it could be task goal related. Seifert and Davids then examined the role of dynamic interaction between intentions, actions and perceptions to influence movement stability in response to changing performance contexts. Using examples from ice climbing and swimming, the authors provide an interesting discussion on how individuals demonstrate stable and variable movement patterns to achieve a functional outcome. To complete the stable of papers in this section, Hristovski and colleagues used examples from rugby union and martial arts to exemplify how a nonlinear pedagogical approach can provide the setting for learners to explore and find atypical creative movement solutions to solve movement problems. The discussion shared by Hristovski and colleagues centers on the close link between the performer and the environment in better understanding how novel movement solutions can emerge in sports contexts. The detailed and comprehensive insights shared in this section nicely captured some of the interesting empirical findings to support why nonlinear learning is possible.

In the last section of this special issue, practical implications relating to coaching and pedagogy are presented. Schöllhorn and colleagues exemplified a 'Differential Learning' approach that is based on the tenets of nonlinear learning to encourage individual learning. Using the acquisition of shooting in football as a research task, the authors shared how a differential learning approach where high movement 
variability is encouraged during practice can lead to effective learning. Renshaw and colleagues provided further discussion to examine how a nonlinear pedagogical approach can assist in increasing motivation and contribute positively to emotional health for learners. The authors attempted to show how nonlinear pedagogy can support the three basic psychological needs that underpin self-determined motivation and provide a useful discussion on some potential practical implications. To round off the last section, Hopper shares how learning is emergent and uses interesting and relevant examples in creative dance and inventing games within a Physical Education context to illustrate the relevance of complexity theory in underpin learning. Realistic examples are discussed and this provides practitioners with some ideas on how a nonlinear pedagogy approach can be actualized in a school setting. Collectively, these papers present a strong understanding on 'when and where' to provide the features of nonlinear learning.
The special issue concludes with a commentary by Davids and colleagues on the work by Newell and Liu. The commentary discusses the importance of creating representative learning environments to ensure that learners become attuned to the critical features inherent in a real game setting. Therefore, practices need to be realistic to help learners manage the critical intertwined relationships between intentions, actions and perceptions present in sports.

This special issue on the nature of nonlinear learning aims to provide researchers and practitioners a starting point to understand and examine the dynamic complexities of skill acquisition from a nonlinear perspective. Importantly, we acknowledge that the content shared here is not exhaustive but hopefully, it can provide valuable insights to 'how' we go about understanding the nature of nonlinear learning. 